\title{
Screening the Presence of Amflora Transgenic Potato in Food or Feed Products
}

\author{
Curticăpean Manuela-Claudia* \\ Cell Biology and Microbiology Department, University of Medicine and Pharmacy, Romania
}

Objective. Amflora potato, event EH92-527-1 was genetically modified to produce only the amylopectin component from starch composition. The presence of the transgenic potato in the food on Romanian market should be verified although the Amflora potato uses for industrial purposes and animal feed. The aim of this study was to detect the presence of the Amflora potato in the food or feed products. Methods. For this purpose, five samples of potato tubers and four samples of chips were analyzed. DNA isolation was performed with E.Z.N.A. ${ }^{\circledR}$ MicroElute Genomic DNA kit (Omega Bio-Tek, USA). For identification a potential presence of the recombinant DNA in the food samples it was used GMOldent RT Event EH92-527-1 Potato kit (Eurofins GeneScan, Germany), that is a real-time PCR kit for qualitative event-specific detection of AmfloraTM EH92-527-1 potato. Results. Amflora transgenic potato was not detected in any of analyzed samples. Conclusions. The results of real-time PCR method confirm the absence of Amflora event EH92-527-1 in all potato tubers and chips analyzed samples.

Keywords: Amflora, screening, real-time PCR, food products

Received 21 October 2016 / Accepted 28 November 2016

\section{Introduction}

On the EU market, the deliberate release into environment of genetically modified organisms (GMOs) and its control and monitoring are submitted to the EU legislation (2003/18/EC Directive). In order to guarantee the protection of the human health and the environment, EU legislation (Regulation No. 1829/2003 and No. 1830/2003) impose GMO labelling if genetically modified (GM) material is present in a product in a proportion above $0.9 \%$ on approved ingredient level $[1,2]$. Regulation No. $1829 / 2003$ does not require labelling of products obtained from animals fed with GMO (meat, milk or eggs) [3].

Conventional potato (Solanum tuberosum L.) is tetraploid and contains two forms of starch: 20\% amylose and 80\% amylopectin and 4 gbss genes. When gbss genes are expressed, the GBSS enzyme (granule bound starch synthase) is synthesized and the amylose is produced [4].

Amflora, event EH92-527-1 (GMO Unique Identifier BPS-25271-9) is a transgenic potato developed by BASF Plant Science company (Germany). Amflora, GM potato, obtained the authorization for cultivation from the European Commission, to be used for industrial purposes (paper industry, textile industry, in adhesives and in construction materials), animal feed (e.q. pulp) or for other conventional non-food purposes (e.q. potato juice used as soil fertilizer). The Amflora tubers are not fit for direct human consumption $[4,5,6,7,8,9,10]$.

Amflora potato was generated through Agrobacteriummediated transformation of the potato cultivar Prevalent [10]. Because amylopectin is the required starch component for industrial applications, Amflora was genetically

* Correspondence to: Manuela-Claudia Curticăpean

E-mail: manuela.curticapean@umftgm.ro modified in starch composition to stop the amylose production and to produce only the amylopectin component $(100 \%)$. When GBSS enzyme (necessary for amylose production) missing, only the amylopectin is produced. In the Amflora potato was inserted an additional copy of the gbss genes in the anti-sense orientation (ssbg genes) that makes to shut down production of the GBSS enzyme. The nptII antibiotic resistance gene (for kanamycin and neomycin) was also introduced in the Amflora potato. This gene is used as a selectable marker for identifying transformed plants $[4,5,6]$. The Amflora potato is only cultivated in order to be used in non-foodstuffs $[4,11]$.

The purpose of this study was to monitor the presence of the Amflora potato in the food or feed products on the market.

\section{Methods}

The samples analyzed in this study were from different Romanian markets. Five samples of potato tubers (autochthonous and imported) and four samples of chips (autochthonous and imported) were analyzed. Samples of potato tubers were labelled C1-C5 and samples of chips were encoded C6-C9.

DNA isolation was performed with E.Z.N.A. ${ }^{\circledR}$ MicroElute Genomic DNA kit (Omega Bio-Tek, USA) using 100 $\mathrm{mg}$ of each sample. DNA isolation procedure was done according to the producer recommendation. E.Z.N.A. ${ }^{\circ}$ MicroElute Genomic DNA kit uses the reversible binding properties of the $\mathrm{HiBind}^{\oplus}$ matrix, a silica-based material, in combination with the MicroElute spin column technology to allow elution volume as small as $10 \mu \mathrm{L}$ [12]. DNA extraction method is composed of four main successive steps: samples lysis under denaturing conditions; DNA bind with MicroElute DNA Mini Columns; DNA purification 
using three quick wash steps; high-quality DNA elution using Elution Buffer [12].

For identification a potential presence of the recombinant DNA in the food samples, GMOIdent RT Event EH92-527-1 Potato kit (Eurofins GeneScan, Germany) it was used. GMOIdent RT Event EH92-527-1 Potato kit is a real-time PCR (polymerase chain reactions) kit for qualitative event-specific detection of Amflora ${ }^{\mathrm{TM}}$ EH92-527-1 potato. The method utilized in this kit, uses the GMOPCR system described in the method published and validated by the CRL (Community Reference Laboratory) of the European Commission [13,14].

PCR was performed in a $25 \mu \mathrm{L}$ volume containing master mix RT (LR) GS-P-09.062 (composed of BasicMix QL RT (LR/HR+) and OligoMix QL RT GS-P-09.062) and target genomic DNA for each test. All samples were compared with duplicates positive and negative control samples [13]. The DNA amplifiability was verified using inhibition control for each sample. Real-time PCR analysis were carried out in a IQ ${ }^{5}$ thermocycler (Bio-Rad). The real-time PCR amplification conditions were: $10 \mathrm{~min}$. at $95^{\circ} \mathrm{C}$ and 45 cycles of $15 \mathrm{sec}$. at $95^{\circ} \mathrm{C}$ and $90 \mathrm{sec}$. at $60^{\circ} \mathrm{C}$ [13].

\section{Results}

Real-time PCR analysis of nine analyzed samples (potato tubers and chips) showed the absence of transgenic potato (Amflora) in all investigated food or feed products. The recombinant DNA was not detected in any samples, as shown in the amplification chart (Figure 1).

Positive signals present in the amplification chart correspond to inhibition control samples and positive control samples (Figure 1, Table I).

\section{Discussion}

Using negative control (NTC) samples in a real-time PCR analysis is needed. Results obtained for NTC samples (Table I) indicate that no contamination of DNA has occurred during pipetting of PCR reactions and the PCR reagents worked properly, not giving false-positive results [13].

Inhibition control samples verify the amplifiability of the DNA [13]. The PCR results for all inhibition control samples investigated were not negative (Figure 1, Table I), so the inhibitory substances are not present in potato and chips samples.

Amflora transgenic potato was not detected in any of analyzed samples as confirmed by results from Figure 1 and Table I.

Such monitoring studies regarding the GMO presence in food products, with negative results were made by a group of researchers from Italy [5].

Continuous monitoring of GMO existing products on the market is necessary for several reasons. First, to check if the EU legislation on labelling produts containing GMOs is respected. Second, due to imported products from coun-

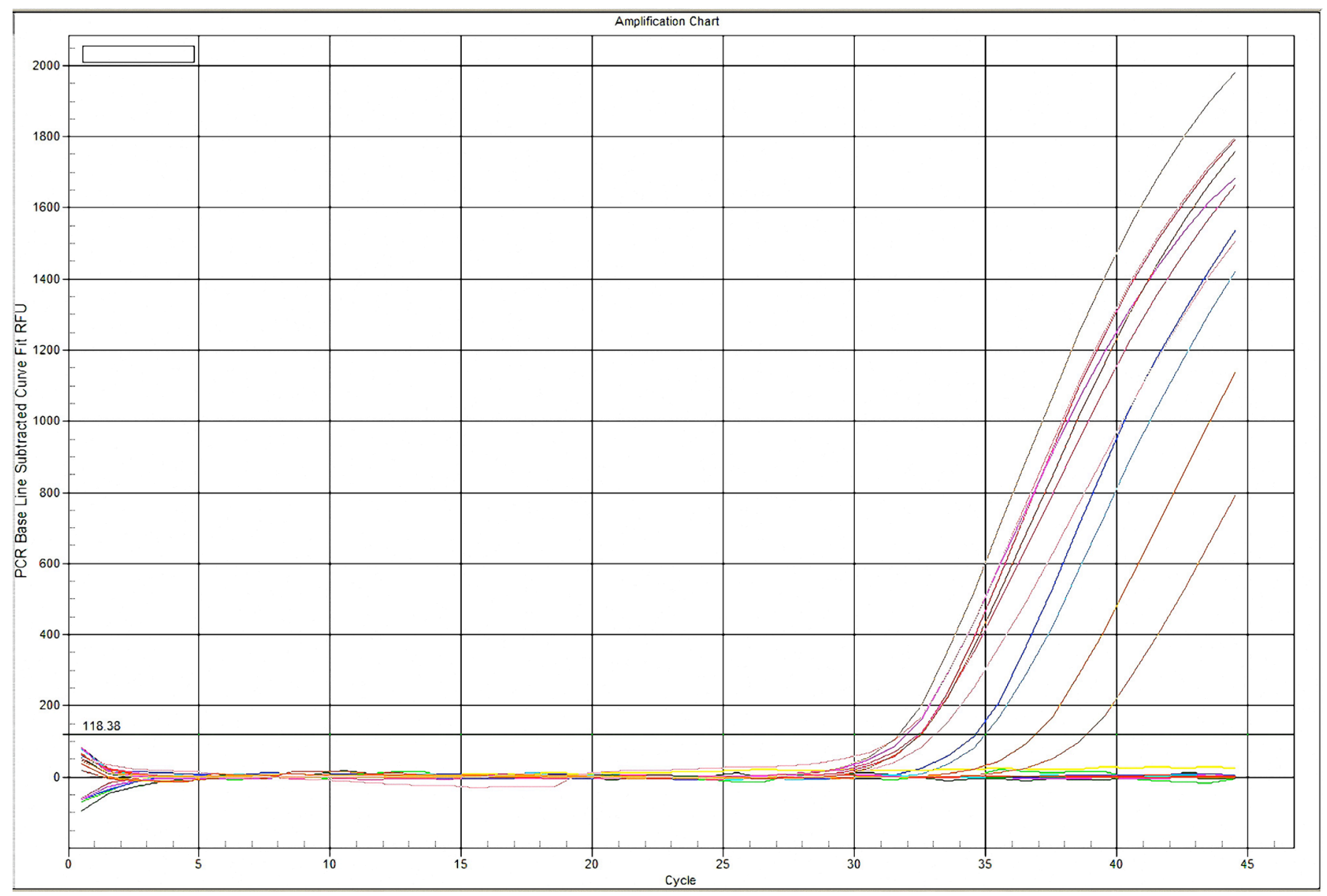

Fig. 1. Amplification chart of the real-time PCR analysis in all nine samples 
Table I. Results of real-time amplification PCR in all analyzed samples

\begin{tabular}{lccc}
\hline Samples & Type & Replicate & Threshold Cycle (Ct) Mean \\
\hline Negative control & NTC & 1 & 00.00 \\
Negative control & NTC & 2 & 00.00 \\
Positive control & Pos Ctrl & 1 & 34.97 \\
Positive control & Pos Ctrl & 2 & 34.60 \\
Tuber potato & C1 & 1 & 00.00 \\
Tuber potato & C2 & 2 & 00.00 \\
Tuber potato & C3 & 3 & 00.00 \\
Tuber potato & C4 & 4 & 00.00 \\
Tuber potato & C5 & 5 & 00.00 \\
Chips & C6 & 6 & 00.00 \\
Chips & C7 & 7 & 00.00 \\
Chips & C8 & 8 & 00.00 \\
Chips & C9 & 9 & 00.00 \\
Tuber potato & C1+ & 10 & 32.45 \\
Tuber potato & C2+ & 11 & 31.95 \\
Tuber potato & C3+ & 12 & 32.38 \\
Tuber potato & C4+ & 13 & 33.03 \\
Tuber potato & C5+ & 14 & 31.77 \\
Chips & C6+ & 15 & 32.51 \\
Chips & C7+ & 16 & 36.87 \\
Chips & C8+ & 17 & 38.81 \\
Chips & C9+ & 18 & 31.66 \\
\hline C1+ C9+ nin sam & &
\end{tabular}

$\mathrm{C} 1+$ - C9+ - nine samples with inhibition control (spiked samples: +)

tries that do not have regulation to present GMOs content on labelling (eq. USA).

Some researchers affirm that the number (number of species; number of countries involved) and the diversity (diversity of novel genetic elements) of commercialized GMOs will increase significantly in the next few years. All of these factors contribute to the increasing complexity of detecting and correctly identifying GMO material $[15,16]$.

Researchers believe that there are several causes for the increasing frequency of unauthorized products on the market. In recent years, more GMOs were developed by Asian technological companies and these GMOs are unlikely to be submitted for EU approval. The possibility that GMOs escape from field-trials, because information about the GMO developments, field-trials and authorizations is often limited in different countries, could be another cause for unauthorized products presence on the market. In this context, researchers used DNA walking methods to identify unauthorized GMOs. These methods allow to rapidly identify the transgene flanking region and involve the use a first reverse primer followed by two semi-nested PCR using primers that are each time nested to the previous reverse primer $[15,16]$.

\section{Conclusions}

The results of real-time PCR method confirm the absence of Amflora, event EH92-527-1 in all potato tubers and chips analyzed samples.

To detect the presence of authorized and unauthorized products on the market, requires a continuous monitoring of these products. In this monitoring process, screening steps, regarding presence or absence of GMOs and quantitative testing to determine the minimum amount of recombinant DNA in samples, are important stages.

\section{Conflict of interest}

None to declare.

\section{References}

1. Regulation (EC) No. 1829/2003 of the European Parliament and of the Council of 22 September 2003 on genetically modified food and feed. Official Journal of the European Union, L268, 1-23.

2. Regulation (EC) No. 1830/2003 of the European Parliament and of the Council of 22 September 2003 concerning the traceability and labelling of genetically modified organisms and the traceability of food and feed products produced from genetically modified organisms and amending Directive 2001/18/EC. Official Journal of the European Union, L268, 2428.

3. Kuiper HA, Davies HV - The Safe Food Risk Analysis Framework suitable for GMOs? A case study. Food Control. 2010; 21:1662-1676.

4. VIB (Vlaams Instituut voor Biotechnologie - Flemish Institute for Biothenology) - Scientific background report Amflora potato. https:// www.vib.be/en/news/Documents/VIB\%20Background\%20report\%20 Amflora.pdf.

5. Tilocca MG, Serratrice G, Oggiano MA et al - Monitoring the presence of genetically modified potato EH92-527-1 (BSP-25271-9) in commercial processed food. Ital J Food Safety. 2014; 3(1): 57-59.

6. Abdallah $\mathrm{N}$ - Amflora great expectations for GM crops in Europe. GM Crops. 2010; 1:3:109-112.

7. European Commission (EU) 2010/135/EU, 2010a. Commission Decision concerning the placing on the market, in accordance with Directive 2001/18/EC of the European Parliament and of the Council, of a potato product (Solanum tuberosum L. line EH92-527-1) genetically modified for enhanced content of the amylopectin component of starch. In: Official Journal of the European Union, L53, 04/03/2010.

8. European Union Reference Laboratory for GM Food and Feed, EU Database of Reference Methods for GMO Analysis. http://gmo-crl. jrc.ec.europa.eu/gmomethods/entry?db=gmometh\&id=qt-eve-st001\&rq=id\%3aQT-eve-ST*

9. Center for Environmental Risk Assessment, GM Crop Database. http:// cera-gmc.org/GmCropDatabaseEvent/EH92-527-1

10. Opinion of the Scientific Panel on Genetically Modified Organisms on a request from the Commission related to the notification (Reference C/SE/96/3501) for the placing on the market of genetically modified potato EH92-527-1 with altered starch composition, for cultivation and production of starch, under Part C of Directive 2001/18/EC from BASF Plant Science. The EFSA Journal. 2006; 323: 1-20. http://onlinelibrary. wiley.com/doi/10.2903/j.efsa.2006.323/epdf

11. European Commission (EU) 2010/136/EU, 2010b. Commission Decision authorizing the placing on the market of feed produced from genetically modified potato EH92-527-1 (BPS-25271-9) and the adventitious and technically unavoidable presence of the potato in food and other feed products, under Regulation (EC) No. 1829/2003 of the European Parliament and of the Council. In: Official Journal of the European Union, L53, 04/03/2010.

12. E.Z.N.A.® MicroElute Genomic DNA kit manual, Omega Bio-Tek, 2013. www.omegabiotek.com

13. GMOldent RT Event EH92-527-1 Potato kit manual, Eurofins GeneScan $\mathrm{GmbH}, \mathrm{v}$, 2016.

14. Event-specific method for the quantification of amylopectin potato event EH92-527-1 using real-time PCR, Protocol EH92-527-1, CRLVL09/05VP, Validation Report EH92-527-1 potato, CRLVL09/05VR, Community Reference Laboratory for GM Food and Feed. EH92-527-Validated_ method corrected version 1.pdf

15. Fraiture M-A, Herman P, Taverniers I, De Loose M, Deforce D, Roosens $\mathrm{NH}-$ An innovative and integrated approach based on DNA walking to identify unauthorized GMOs. Food Chem. 2014; 147: 60-69.

16. Holst-Jensen A, Bertheau Y, de Loose M et al - Detecting un-authorized genetically modified organisms (GMOs) and derived materials. otechnology Advances. 2012; 30: 1318-1335. 